\title{
TTR
}

Traduction, terminologie, re?daction

\section{Histoire et didactique de la traduction}

\section{Michel Ballard}

Volume 8, numéro 1, 1er semestre 1995

Orientations européennes en traductologie

URI : https://id.erudit.org/iderudit/037203ar

DOI : https://doi.org/10.7202/037203ar

Aller au sommaire du numéro

\section{Éditeur(s)}

Association canadienne de traductologie

ISSN

0835-8443 (imprimé)

1708-2188 (numérique)

Découvrir la revue

\section{Citer cet article}

Ballard, M. (1995). Histoire et didactique de la traduction. TTR, 8(1), 229-246. https://doi.org/10.7202/037203ar

\section{Résumé de l'article}

Histoire et didactique de la traduction - Selon l'auteur, la traduction ne peut devenir une discipline autonome dans le cadre universitaire que si elle fonde son identité sur la traductologie et la didactique. Le problème qui se pose dès lors est celui de la formation des enseignants et du contenu didactique de la traduction. Cet article explore la didactique de la traduction sous l'angle historique en passant en revue un certain nombre de précurseurs: notamment Gaspard de Tende (1660), Ferri de Saint-Constant (1811) renvoyant aux auteurs de l'Antiquité, Charles Rollin (1838)... Et l'auteur se demande comment il se fait que les didacticiens du XXe siècle aient eu l'impression que tout commençait avec eux, quand en réalité c'est à une renaissance que l'on assiste depuis les années 1950 (la Stylistique comparée du français et de l'anglais publiée en 1958 constituant l'événement symbolique de cette renaissance) beaucoup plus qu'à une véritable naissance.
Tous droits réservés @ C TTR: traduction, terminologie, rédaction — Les auteurs, 1995 document est protégé par la loi sur le droit d'auteur. L’utilisation des services d'Érudit (y compris la reproduction) est assujettie à sa politique d'utilisation que vous pouvez consulter en ligne.

https://apropos.erudit.org/fr/usagers/politique-dutilisation/ 


\section{Histoire et didactique de la traduction}

\section{Michel Ballard}

\section{Introduction}

Dans un article récemment paru dans cette revue, Jean Delisle (1992) déplorait l'absence d'homogénéité de nombreux manuels de traduction (p. 23); ce phénomène étant, selon lui, lié au fait qu'ils ne sont pas toujours conçus pour un public précis, avec des objectifs bien spécifiques: «Plusieurs manuels s'adressent à un public très large et souvent mal défini» (p. 22). Ce défaut, à notre sens, est tout autant dû aux mots d'ordre des éditeurs (ou des directeurs de collection) qui, pour des raisons commerciales évidentes, visent à toucher le public le plus large possible, qu'à l'absence de statut officiel de la didactique de la traduction dans l'institution pédagogique. Il y a eu une prise de conscience de cet état de fait chez certains universitaires. Par exemple dans l'avant-propos de leur manuel de Versions Anglaises, A. Castagna et al. (1971, p. iii) déclarent:

La version anglaise a eu pendant longtemps un statut incertain. Tantôt servante de la littérature (explication de texte du type oral d'agrégation), tantôt son égale (certificat $L$ ), elle s'est trouvée aussi associée à la civilisation (certificat d'études pratiques). Elle devrait être aujourd'hui une discipline autonome à tous les niveaux. (Nous soulignons.) 
Or il est bien évident que si la traduction (et pas simplement la version) veut devenir une discipline autonome dans le cadre universitaire, elle ne pourra le faire qu'en fondant son identité sur la didactique et la traductologie. Tant que la traductologie n'aura pas de statut autonome dans le cadre de l'Institution universitaire, les TD de traduction demeureront ce qu'ils sont souvent: des séances aux objectifs assez flous, pour lesquelles tout le monde se sent plus ou moins qualifié. Très curieusement, ce sont les professionnels qui, malgré leur maîtrise du métier et malgré les finalités pratiques de leur enseignement, ont souvent été parmi les premiers à prendre conscience de la nécessité de structurer leur enseignement à l'aide d'une forme de théorisation. Nous renvoyons sur ce point au témoignage de Christine Durieux (1988) à propos de ses débuts comme enseignante à l'ESIT; il a une valeur très générale et s'applique aisément au domaine de la «traduction pédagogique»:

Le jeune professeur d'histoire ou de géographie qui se présente pour la première fois devant ses élèves dispose déjà d'un cadre de travail qui dans un premier temps va le sécuriser, c'est le programme; il doit suivre le programme. C'est le cas pour tous ceux qui sont chargés de transmettre un savoir. Dans le cas de la traduction, il n'existe rien de tel [...]. (p. 8)

Cette constatation pose le problème de la formation des enseignants et du contenu de la didactique de la traduction. Celui-ci ne saurait se limiter (s'il veut être réaliste), à des leçons de linguistique contrastive, même si cette composante peut se révéler fort utile et productive dans le cadre général de la traductologie. La composante que nous nous proposons d'explorer dans cet article est la dimension historique dans son rapport à une didactique de la traduction: comme antériorité d'une existence, comme fondement de certaines démarches, enfin comme objet d'étude et comme ouverture.

\section{Des précurseurs}

En 1960, Georges Mounin (1976) saluait la Stylistique comparée de Vinay et Darbelnet en ces termes: 
Il ne manque pas d'ouvrages sur la traduction. La bibliographie fondamentale, qui n'existe pas encore, en serait fort longue. Mais cet ouvrage est sans doute le premier traité de traduction. (p. 227)

Il s'agit d'un livre neuf, qui donne de bonnes descriptions des opérations de traduction, puis un classement - dont le détail est parfois peu clair, estompé par une poussière d'observations; mais c'est le premier! (pp. 228-229)

Il est vrai qu'à l'époque l'ouvrage apparaissait comme neuf, et même révolutionnaire, surtout aux yeux d'étudiants (dont nous étions) qui pour la première fois se trouvaient confrontés avec une présentation ordonnée de problèmes jusque-là rencontrés dans le désordre, au hasard de la traduction de textes, et sans même toujours être identifiés. Car il faut décrire et nommer pour identifier; une prise de conscience intuitive est toujours très lente, et l'on ne peut demander à chaque individu du $\mathrm{XX}^{\mathrm{e}}$ siècle de refaire tout seul le chemin intellectuel que des générations ont parcouru avant lui; c'est là sans doute l'erreur des adversaires de la théorisation; ils freinent l'accès à la réflexion, à une perception plus rapide des problèmes.

Mais à y regarder de plus près, l'impression que décrit Mounin n'est-elle pas inquiétante? Il a fallu attendre une étape bien avancée de l'Homo erectus (entre 400000 et 300000 ) pour que se mettent en place les organes permettant le langage articulé'. L'écriture n'apparaît qu'au quatrième millénaire av. J.-C. et les traces les plus anciennes que l'on possède concernant les langues attestent leur diversité ${ }^{2}$ et donc la nécessité de leur apprentissage et de la traduction. On a des traces attestant une activité de traduction dès le troisième millénaire avant notre ère ${ }^{3}$. Au IV siècle av. J.-C.

1. Voir, entre autres, Hagège (1985), chapitre I, «Unicité de l'espèce, pluralité des langues», p. 14 sqq.

2. Ibid. et Vendryes (1979), «Introduction: l'origine du langage», p. 17 sqq.

3. Cf. Ballard (1992a), pp. 21-22 pour l'Égypte, p. 24 pour Sumer. 
les Grecs entament une forme de réflexion sur le langage, qui sera reprise par les Romains et servira de base aux études pendant tout le Moyen Âge, et même au-delà. À côté de cela, la traduction fait figure de parent pauvre: activité secondaire (ou considérée, à tort, comme allant de soi), elle n'a pas donné lieu à des traités comme les genres littéraires «nobles». Car c'est de cela qu'il s'agit en fait dans la citation ci-dessus; Mounin tout en reconnaissant l'existence d'écrits sur la traduction déplore l'absence de traité, c'est-à-dire d'étude systématique axée sur le sujet. Or, même là, on peut se demander, avec tout le respect dû à son œuvre magistrale, à sa perspicacité et à son sens de la mesure, si Georges Mounin n'est pas sous l'influence des «préjugés» de son époque: on connaît alors mal l'histoire de la traduction, encore moins celle de la didactique de la traduction; et il est vrai, il faut le répéter, que l'ouvrage de Vinay et Darbelnet donnait le sentiment d'être une nouveauté dotée d'une épaisseur et d'une densité que n'avaient pas les rares vade-mecum existant alors sur la question. Nous pensons à des ouvrages tels que ceux de Veslot et Banchet (1928) et de Veslot (1928) encore largement diffusés et utilisés dans les années soixante; il n'y a bien entendu aucun mépris dans cette comparaison, simplement le souci de parcourir et de baliser un domaine. Ces ouvrages ont (fort bien) rempli leur fonction en leur temps. Mais n'y avait-il vraiment rien d'autre avant? Il nous semble que cette interrogation mérite d'être posée d'un point de vue général d'abord, celui de la simple curiosité intellectuelle; du point de vue de l'histoire de la didactique des langues ensuite, parce qu'il nous semble normal en ce domaine, comme dans d'autres, de se demander s'il n'y a pas eu des précurseurs et où se situe le commencement. Dans le cadre de cet article nous ne pouvons qu'évoquer un jalon et inviter à poursuivre des recherches dans ce domaine.

En 1811, Ferri de Saint-Constant (dont des extraits ont paru dans D'hulst, 1990) déplore:

[...] il est surprenant que les Latins, qui ont tout emprunté des Grecs, et qui les ont si souvent imités et copiés, n'aient rien écrit sur cette matière [l'art de traduire]. Nous ne connaissons que deux passages de Cicéron et d'Horace où il est question de traduire. 
Quintilien, dont l'ouvrage est si étendu et complet, ne met pas la traduction des grands modèles au nombre des moyens propres à former un orateur ${ }^{4}$.

S'il est faux de dire que Quintilien «ne met pas la traduction des grands modèles au nombre des moyens propres à former un orateur $\left.{ }^{5}\right\rangle$, il est vrai par contre que l'on a l'impression d'une certaine absence de théorisation dans l'antiquité gréco-latine ${ }^{6}$. Pourquoi? Nous évoquions ci-dessus deux pistes possibles: le fait que la traduction est une activité secondaire et celui qu'elle semble «naturelle», aller de soi; il nous semble qu'il convient d'y ajouter une autre raison, qui est de taille et qui, d'ailleurs, de façon logique est très étroitement liée au développement que connaissent les études traductologiques aujourd'hui, à savoir la domination d'une langue et d'une culture.

L'Antiquité a connu deux formes successives d'hégémonie linguistico-culturelle: celles du grec et du latin. On peut presque dire que la civilisation grecque ignorait superbement ses voisines qu'elle considérait comme barbares et la conséquence a été une absence de traductions et donc une absence de réflexion sur une activité qui était niée. La traduction est une forme de négociation entre les langues et les cultures; les problèmes d'identité (et pas seulement celle du texte) et d'hégémonie sont essentiels aux formes qu'elle prend: depuis le gommage de l'altérité jusqu'à son intégration. À la traduction se trouvent liées la curiosité et l'ouverture à l'étranger; ce n'est pas un hasard sans doute si les Grecs du Péloponèse n'ont pas

4. De Saint-Constant (1811), p. 187, cité par D’hulst (1993), p. 105.

5. Quintilien dit: «c'est [la traduction] le moyen le plus sûr pour acquérir l'abondance et la facilité d'élocution. Nos anciens orateurs ne connaissaient rien de mieux pour cela, que de traduire du grec au latin», De Institutione Oratoria, in Horguelin (1981), p. 21 .

6. Cette absence doit être nuancée; nous renvoyons sur ce point au chapitre I de notre ouvrage (1992a). 
traduit du $\mathrm{V}^{\mathrm{e}}$ au $\mathrm{III}^{\mathrm{e}}$ siècle, alors que la dynastie des Ptolémées installée en Egypte et issue d'un des généraux d'Alexandre commande des traductions, $s$ 'intéresse à des civilisations autres que celle de ses origines. Le périple du Grand Macédonien les avait, par son cosmopolitisme, ouverts à d'autres horizons que ceux du Bosphore.

Le phénomène est légèrement différent pour la civilisation latine dans la mesure où la traduction a été le canal par lequel la littérature grecque a été assimilée et transformée en littérature latine. Il semble que deux attitudes prévalaient: celle de l'assimilation qui tend à gommer les différences et donc les possibilités de comparaison; celle de l'imitation qui permettait de se faire un style à partir de textes prestigieux. C'est de là que l'on peut dater une forme d'utilisation didactique consciente de la traduction. On en trouve le principe général chez Cicéron", et il est repris chez Quintilien et Pline le Jeune, mais sans étude générale du problème. Tout au cours du Moyen Âge et même au-delà, en Occident, l'existence du latin comme langue de culture réduit les besoins en traduction. L'affirmation des langues "vulgaires» à la Renaissance accroît le volume des traductions et déclenche une conscience plus aiguë du phénomène qui donne naissance aux premiers traités.

À l'inverse du monde antique, caractérisé par une hégémonie linguistico-culturelle, l'Europe contemporaine se caractérise "par la reconnaissance officielle de l'altérité et de l'incohérence dans les cultures» (Lambert, 1992, p. 17), et c'est ce respect de la diversité linguistique et culturelle ${ }^{8}$ qui fait que la

7. Rappelons le passage célèbre: «[...] imiter Démosthène, c'est, on le comprend, posséder à la fois l'éloquence attique et l'éloquence parfaite», Cicéron (1921), p. 111.

8. «Depuis que le Conseil a établi l'égalité des langues officielles des États membres de la Communauté européenne en 1958, le multilinguisme constitue un principe fondamental du fonctionnement communautaire», Eduard Brackeniers (1992), p. 17. 
traduction y est un phénomène fondamental et l'objet d'importantes études tant sur le plan fondamental qu'à des fins didactiques ${ }^{9}$. C'est la même logique qui a présidé à la naissance d'une traductologie de tendance linguistique au Canada ${ }^{10}$.

À l'époque où paraît la Stylistique comparée (1958) de Vinay et Darbelnet, la France n'a à offrir que Sous l'invocation de saint Jérôme de Valery Larbaud (1946), les Belles infidèles de Georges Mounin (1955), la Traduction dans le monde moderne d'Edmond Cary (1956). On s'étonnera peut-être de l'utilisation d'une locution restrictive pour parler de trois ouvrages relativement prestigieux, mais il convient de prendre conscience que leur présence dessine un manque que précisément celui de Vinay et Darbelnet viendra combler. L'ouvrage de Larbaud est à peine un livre, c'est un collage hétéroclite, une série d'essais, parfois techniques, parfois lyriques, parfois même sans rapport avec la traduction; mais l'ensemble n'est pas construit, on est encore dans la tradition de l'essayisme littéraire, aimable mais a-théorique. L'ouvrage de Cary représente un remarquable effort d'un traducteur pour donner, comme l'indique le titre, un panorama de sa profession dans le monde moderne; il y a aussi des aperçus historiques intéressants et

9. Voir par exemple Seleskovitch et Lederer (1984 et 1989), Durieux (1988) et d'autres travaux venus d'Angleterre - comme ceux de Newmark.

10. En contrepoint, on notera le développement relativement faible de ce genre d'études aux États-Unis. Dans cet esprit, Edwin Gentzler, à propos de «The American Translation Workshop», note: «Today, while many universities offer advanced degrees in creative writing, comparatively few offer academic credit for literary translation. One reason is surely the monolinguistic nature of the culture [...]. The activity of translation represents a process antithetical to certain reigning literary beliefs, hence its relegation to marginal status within educational and economic institutions, and its position in this society as part of a counter-cultural movement» (Gentzler, 1993, p. 8). Nous renvoyons sur ce dernier point à l'ouvrage de Brisset (1990). 
des réflexions très perspicaces mais un refus très net d'envisager l'opération de traduction dans le cadre d'une systématique. L'ouvrage de Georges Mounin a sans doute eu la malchance d'avoir été baptisé d'un titre séduisant mais trompeur par un éditeur qui était plus soucieux de rechercher un titre accrocheur que d'annoncer le véritable contenu de l'œuvre; on y trouve en fait l'esquisse d'une théorisation qui s'épanouira dans les Problèmes théoriques; la caractéristique et la différence des Belles infidèles étant qu'elles ne sont pas encore coupées des racines historiques de la théorisation et que la théorisation qu'on y trouve se préoccupe davantage des «manières de traduire» que les Problèmes théoriques. Les Belles infidèles apparaissent alors comme les prolégomènes à une dissection de la traduction, qui nous est venue du Canada. L'option didactique de Vinay et Darbelnet les amenait à redonner à la théorie son véritable sens: être une observation qui permette de conceptualiser les démarches d'une opération et donc facilite la gestion (et la transmission) de sa pratique. Il faut pour cela passer à l'observation de la traduction comme produit de deux langues-cultures.

Or, cet effort de théorisation avait été réalisé auparavant, et qui plus est en liaison avec la didactique des langues. Les Règles de la traduction de Gaspard de Tende ${ }^{11}$ sont du Vinay et Darbelnet avant la lettre. La qualité de l'ouvrage est encore attestée au milieu du XVIII siècle par l'abbé Goujet dans son examen «des traités sur la manière de traduire» $(1751$, p. 207): «son traité de la traduction, est le meilleur ouvrage et le plus complet que nous ayons en François sur cette matière». L'ouvrage de Gaspard de Tende a inspiré d'autres didacticiens du XVII ${ }^{e}$ siècle:

Le traité de $\mathrm{M}$. de Tende est presque l'unique fonds où ont puisé Jean Gaillard, qui donna en 1673 une Méthode facile et curieuse pour la traduction; et Denys Gaullyer, depuis professeur au Collège du Plessis à Paris, qui publia en 1719, un petit livre de

11. Pour une étude détaillée de ce manuel, nous renvoyons à notre ouvrage (1992a), pp. 186-195, ainsi qu'à M. Ballard, in M. Ballard et $L$. D'hulst (dir.), à paraître. 
Règles pour traduire le Latin en François. (C.P. Goujet, 1751, p. 209)

Le XVIII ${ }^{e} s$. a connu un indéniable essor en matière de réflexion sur la traduction et une poursuite fort active de l'œuvre didactique inaugurée par un de Tende.

Or, pourquoi au milieu du $\mathrm{XX}^{\mathrm{e}}$ siècle a-t-on eu l'impression non pas d'un tarissement de la didactique mais d'une absence (attestée, nous l'avons dit plus haut, par la manière dont l'ouvrage de Vinay et Darbelnet a été salué)? Ce silence mériterait une étude et justifie la mise en place d'une recherche sur l'histoire de la didactique de la traduction: un futur enseignant se doit de réfléchir à la matière qu'il va enseigner et cette réflexion doit prendre en compte le passé de cette discipline.

\section{Du commentaire de traduction}

Dans le prolongement de cette prise en compte du passé afin d'y rechercher les origines de démarches contemporaines ou les phases de rupture, nous examinerons maintenant un aspect essentiel de la traduction qui est sous-exploité en didactique malgré, une fois encore, des tentatives antérieures dignes d'intérêt.

Une théorie comme celle de Seleskovitch et Lederer met l'accent sur l'exégèse; elle s'inscrit en cela dans la tradition inaugurée par les traducteurs bibliques, dont saint Jérôme. Jérôme fait précéder son travail de traduction, ou dans certains cas l'accompagne, d'une œuvre de commentateur. Pour les épîtres de Paul, sa méthode est la suivante: quelques lignes du texte latin, puis le corps du commentaire où non seulement il interprète le sens mais retourne à l'original hébreu; il va jusqu'à fournir deux interprétations ou plus, en laissant le lecteur libre de choisir celle qui lui convient (Kelly, 1975, pp. 144-147). Il est capital que son travail de retraduction de l'Ancien Testament se soit appuyé sur la lecture de l'Hexaples d'Origène, qui repose sur le principe de la comparaison des textes et du commentaire de traduction. L'Hexaples, que Jérôme pouvait consulter à la bibliothèque de 
Césarée, offrait sur six colonnes parallèles: le texte hébreu, une translittération en grec, la version d'Aquila, celle de Symmaque, la Septante et enfin la version révisée de Téodotion; chose remarquable la cinquième colonne constituait à elle seule une édition critique dans la mesure où elle signalait les différences avec le texte hébreu. Le travail de Jérôme qui a abouti à la Vulgate repose donc sur l'exégèse et le commentaire de traduction.

Les principes de la comparaison de traductions et du commentaire de traduction sont à la base de la méthode de Gaspard de Tende, ce sont eux qui lui donnent son caractère scientifique. Voici en quels termes l'auteur décrit la genèse de son œuvre:

Ce qui me donna la première pensée de recueillir ces Règles, fut l'accord merveilleux et la convenance admirable qui se rencontre dans tous les bons Traducteurs. Car j'ay remarqué que ceux qui ont bien traduit les mêmes mots et les mêmes phrases, ont tous pris un même tour, et se sont tous servis d'une même façon de traduire (G. de Tende, 1660, II) ${ }^{12}$.

Nous dirions aujourd'hui que le travail de de Tende repose sur l'observation des récurrences. Mais ce n'est pas le seul usage qu'il fait de la comparaison; il l'utilise également à des fins d'exposé didactique en proposant d'autres solutions (parfois parce qu'elles sont moins bonnes) au jugement de ses lecteurs.

Dans l'exemple suivant, on a une illustration de ce procédé de présentation et de formation du bon goût par rapport à une norme (celle de l'embellissement et de l'autorité de Vaugelas):

At Regina gravi jamdudum saucia cura Vulnus alit venis, et caero carpitur igni.

12. La préface de l'original n'est pas paginée; c'est nous qui introduisons la traditionnelle pagination des préfaces en chiffres romains par opposition à la pagination du corps de l'ouvrage. 
Mais Didon qui estoit déjà troublée par des cuisantes inquiétudes, cache une blessure secrète dans le secret de son coeur, et se sent dévorer d'un feu qu'elle ne connoit point encore. Virg.

Autre traduction de ces deux vers:

La Reine agitée depuis long-temps d'une violente inquiétude nourrissait sa playe dans ses veines, et sentoit son âme éprise d'un feu secret.

Cela fait bien voir qu'il faut souvent traduire le nom Appellatif par le nom propre; afin de garder quelque grace dans la traduction: ce qui est tellement nécessaire que monsieur de Vaugelas en a ainsi usé dans son Q. Curce (G. de Tende, 1660, p. 16).

Au siècle suivant, dans son Traité des études (1838) Rollin propose le commentaire de traduction comme exercice à faire réaliser par les étudiants. Il ne s'agit plus d'une démarche de travail scientifique et d'exposition comme chez de Tende mais d'un véritable exercice pédagogique visant à affiner l'esprit critique. Le caractère pédagogique de la démarche de Rollin est de plus attesté dans la progression qu'il instaure. Il commence par proposer un commentaire simple qui fonctionne comme une sorte de travail d'auto-correction où l'élève est invité à trouver ses erreurs et ses insuffisances par rapport à un modèle prestigieux et à les commenter:

Il n'est pas inutile, quand on aura ces auteurs traduits par une main savante, de comparer cette traduction avec celles des écoliers, pour leur donner du courage, et leur proposer de bons modèles. Il ne rougiront point d'être vaincus par de tels maîtres; ils tiendront à honneur de les suivre, quoique de loin; etc. (Ch. Rollin [1726-1730] 1838, pp. 119-120).

et il ajoute après avoir donné un exemple de commentaire:

Il me semble que cette sorte de critique peut être utile aux jeunes gens, et que c'est un bon moyen pour leur former le jugement, que de leur proposer des difficultés comme j'ai fait ici, et de 
tâcher de leur en faire trouver à eux-mêmes la solution, si cela est possible (ibid., p. 126).

Voici enfin ce qu'il dit du commentaire comparé:

Il me semble que c'est un exercice fort utile, que de faire ainsi de temps en temps comparer aux jeunes gens deux traductions d'un même endroit, et de leur faire remarquer à eux-mêmes les différences en bien et en mal, surtout après qu'ils l'ont aussi traduit de leur côté. Par là, ils en peuvent mieux sentir et les beautés et les défauts; et ils apprennent ce qu'il faut suivre et éviter pour réussir dans la traduction (ibid., p. 143).

Dans le même esprit, dès 1986 (p. 35 sqq.), nous avons préconisé l'utilisation du commentaire de traduction comme exercice pédagogique complémentaire à ceux du thème et de la version. Nous avons précisé les modalités de sa forme simple dans un article spécifique (1988), pour enfin consacrer un manuel entier à la didactique du commentaire (1992b).

Dans ce dernier ouvrage nous partons du principe que l'observation de la traduction est une démarche pédagogique et scientifique, une voie d'accès à la pratique et à la réflexion. Elle suppose la mise en place de concepts et d'une terminologie précise permettant d'analyser des problèmes, des processus. La technique du commentaire y est envisagée à deux niveaux:

- celui du commentaire simple, qui peut lui-même connaître des degrés: depuis le simple exercice d'initiation ou de contrôle (permettant de vérifier l'acquisition de certaines notions) jusqu'à un exercice plus complexe qui se rapproche des commentaires que l'on trouve dans les manuels de traduction reposant sur des bases théoriques solides ${ }^{13}$,

13. Voir par exemple Chuquet (1990) et Demanuelli (1991), qui offrent des exemples très élaborés de commentaires, éclairant la dynamique de traduction. 
- et le niveau du commentaire comparé, où l'on propose deux traductions, ou plus, d'un même texte. Cet exercice est enrichissant sur le plan de l'observation des techniques de traduction et des tendances propres à chaque langue. C'est une propédeutique à la recherche, une phase de collecte de matériaux préalable à l'élaboration de synthèses sur des thèmes très divers (Ballard, 1992b, pp. 107-110).

\section{Conclusion}

Le développement de la didactique de la traduction peut paraître un phénomène récent. Ce sentiment vient sans doute du développement général des études traductologiques et du désir plus ou moins connexe de nombreux enseignants de renouveler l'enseignement de traduction en le dotant d'une méthodologie propre.

Le développement des études traductologiques en direction de l'histoire amène à prendre conscience du fait que ce sentiment de nouveauté est aussi en grande partie créé par l'ignorance du passé de la traductologie et en particulier de la didactique de la traduction. Des réflexions ont existé antérieurement, des méthodes aussi. On est en droit de s'interroger sur les causes de l'oubli (ou de l'occultation) qui les a marquées. Il y a là une piste de recherche importante et nécessaire.

L'histoire de la traduction et de sa didactique révèle l'importance du commentaire à la fois comme démarche pédagogique et théorico-pratique. Le commentaire précède et accompagne la traduction. On peut en faire un moyen d'observation et d'étude à partir de textes déjà traduits.

Le développement que connaît à l'heure actuelle la didactique de la traduction n'est pas une nouveauté mais une renaissance qui vise à faire de la traduction un acte pédagogique et intellectuel plus complet. Les pédagogues se doivent de renouer avec cette tradition qui est la source de leur identité. 
Michel Ballard: U.F.R. Langues et civilisations étrangères, Université d'Artois, 9 rue du Temple, B.P. 665, 62030 Arras Cedex (France)

\section{Références}

BALLARD, Michel (1986). «Pour un enseignement de traduction», Franco-British Studies, $\mathrm{n}^{\circ}$ 1, printemps, pp. 27-40.

XXXIII(3), pp. 341-349.

(1988). «Le commentaire de version», Meta,

(1992a). De Cicéron à Benjamin. Traducteurs, traductions, réflexions. Lille, PUL, coll. «Étude de la traduction».

(1992b). Le Commentaire de traduction anglaise. Paris,

Nathan.

(à paraître). «Gaspard de Tende, théoricien de la traduction", in M. Ballard et L. D'hulst, dir., la Traduction en France à l'âge classique.

BRACKENIERS, Eduard (1992). «L'Europe et le multilinguisme», in Christine Pagnoulle, dir., les Gens du passage. Liège, Presses de l'Université, pp. 17-20.

BRISSET, Annie (1990). Sociocritique de la traduction. Théâtre et altérité au Québec (1968-1988). Longueuil (Québec), Le Préambule.

CASTAGNA, A., M. CLING, et al. (1971). Versions anglaises. Paris, Hachette, coll. «Hachette Université».

CARY, Edmond (1956). La Traduction dans le monde moderne. Genève, Georg.

CHUQUET, Hélène (1990). Pratique de la traduction. Gap, Ophrys. 
CICÉRON (1921). Du meilleur genre d'orateurs [46 av.J.-C.], texte établi et traduit par Henri Bornecque. Paris, Les Belles Lettres.

DELISLE, Jean (1980). L'Analyse du discours comme méthode de traduction. Ottawa, Les Presses de l'Université coll. «Cahiers de traductologie» $\mathrm{n}^{\circ} 2$.

(1992). «Les manuels de traduction : essai de classification», TTR V(1), pp. 17-47.

DEMANUELLI, Claude et Jean (1991). Lire et traduire, anglaisfrançais. Paris, Masson.

D'HULST, Lieven (1990). Cent ans de théorie française de la traduction. De Batteux à Littré (1748-1847). Lille, PUL.

(1993). "Observations sur l'expression figurée en traductologie française $\left(\mathrm{XVIII}^{\mathrm{e}}\right.$-XIX ${ }^{\mathrm{e}}$ siècles)», TTR $\mathrm{VI}(1)$, pp. 83111.

DURIEUX, Christine (1988). Fondements didactiques de la traduction technique. Paris, Didier, coll. «Traductologie» $n^{\circ} 3$.

FERRI de SAINT CONSTANT, J.L. (1811). Rudiments de la traduction ou l'Art de traduire le latin en français [1808]. Paris, A. Delalain.

GENTZLER, Edwin (1993). Contemporary Translation Theories. Londres et New York, Routledge.

GOUJET, Claude Pierre (abbé) (1751). «Des traités sur la manière de traduire», Bibliothèque française ou histoire de la littérature française. Paris, Guérin-Lemercier, T. 1, pp. 205-219.

HAGÈGE, Claude (1985). L'homme de parole. Paris, Fayard.

HORGUELIN, Paul A. (1981). Anthologie de la manière de traduire. Domaine français. Montréal, Linguatech. 
KELLY, J.N.D. (1975). Jerome. Londres, Duckworth.

LAMBERT, José (1992). «Traduction et institutions», in Christine PAGNOULLE, dir., les Gens du passage. Liège, Presses de l'Université, pp. 21-26.

LARBAUD, Valery (1986). Sous l'invocation de saint-Jérôme [1946]. Paris, Gallimard.

MOUNIN, Georges [1955]. Les Belles Infidèles. Réédité en 1994: Lille, PUL, coll. «Étude de la traduction».

(1960). Compte rendu de J.-P. Vinay et J. Darbelnet, Stylistique comparée du français et de l'anglais (1958), Bulletin de la Société de Linguistique, T. 55, 1960, fascicule 2, pp. 46-50, repris dans Georges MOUNIN, Linguistique et traduction, Dessart et Mardaga, 1976, pp. 227-234.

Paris, Gallimard.

(1963). Les Problèmes théoriques de la traduction.

PAGNOULLE, Christine, dir. (1992). Les Gens du passage. Liège, Presses de l'Université.

QUINTILIEN. Euvres complètes de Quintilien, trad. de la Collection Panckoucke par C. V. Ouizille. Paris, Garnier Frères, vol. III.

RIVARA, Annie (1990). «La Problématique de l'insertion d'un modèle culturel: deux traductions d'Arcadia (1625)", Littératures classiques, 13, pp. 147-162.

ROLLIN, Charles (1838). «De la traduction», Traité des études [1726-1730]. Paris, Saintin et Thomine, T. 1., pp. 115-155.

SELESKOVITCH, Danica et Marianne LEDERER (1984). Interpréter pour traduire. Paris, Didier, «Traductologie» $\mathrm{n}^{\circ} 1$. 
(1989). Pédagogie raisonnée de l'interprétation. Paris, Didier, coll. «Traductologie» $n^{\circ} 2$.

TENDE, Gaspard de, Sieur de l'Estang (1660). Règles de la traduction, ou moyens pour apprendre à traduire de latin en françois. Paris, Damien Foucault.

VESLOT, H. et J. BANCHET (1968). Les Traquenards de la version anglaise [1928]. Paris, Hachette.

VESLOT, H. (1965). Les Épines du thème anglais [1928]. Paris, Hachette.

VINAY, Jean-Paul et Jean DARBELNET (1966). Stylistique comparée du français et de l'anglais [1958]. Paris, Didier.

VENDRYES, Joseph (1979). Le Langage. Introduction linguistique à l'histoire [1923]. Paris, Albin Michel.

RÉSUMÉ: Histoire et didactique de la traduction - Selon l'auteur, la traduction ne peut devenir une discipline autonome dans le cadre universitaire que si elle fonde son identité sur la traductologie et la didactique. Le problème qui se pose dès lors est celui de la formation des enseignants et du contenu didactique de la traduction. Cet article explore la didactique de la traduction sous l'angle historique en passant en revue un certain nombre de précurseurs: notamment Gaspard de Tende (1660), Ferri de SaintConstant (1811) renvoyant aux auteurs de l'Antiquité, Charles Rollin (1838)... Et l'auteur se demande comment il se fait que les didacticiens du $\mathrm{XX}^{\mathrm{e}}$ siècle aient eu l'impression que tout commençait avec eux, quand en réalité c'est à une renaissance que l'on assiste depuis les années 1950 (la Stylistique comparée du français et de l'anglais publiée en 1958 constituant l'événement symbolique de cette renaissance) beaucoup plus qu'à une véritable naissance. 
ABSTRACT: Translation History and Didactics - According to the author, translation can only attain the status of an autonomous discipline within the university if its identity is based on both Translation Studies and Translation Teaching. Hence, the ensuing problem becomes that of teacher training and the didactic content of translation. This article explores translation teaching from an historical perspective, reviewing a few precursors such as Gaspard de Tende (1660), Ferri de Saint-Constant (1811) who refers to authors of Antiquity, and Charles Rollin (1838). The author wonders why twentieth century teaching specialists seem to think that they began it all when in fact since the 1950's we are witnessing a rebirth (la Stylistique comparée du français et de l'anglais published in 1958 was a symbolic landmark of this rebirth) rather than the birth of a trend. (Trans. by Paul Bandia) 